\title{
CS technology information hiding algorithm based on capacity of image block
}

\author{
Peng-Yi Tian and Ding-Gen Xu \\ College of Non-commissioned Officer of Changping,the Academy of Equipment, Beijing \\ Changping, China \\ E-mail: 437613575@qq.com
}

\begin{abstract}
For compressed sensing technology used in the field of information hiding, the traditional algorithm is to measure the entire carrier ( large redundancy - the entire carrier of the measured value) to hide. The calculation time is long and the stego image imperceptibility can be further improved. In this paper, first according to the number of secret information block the carrier and the complexity of vector selection and information hiding based on measurement, experiments show that this algorithm improves the imperceptibility and saves the computing resources. The effect is ideal.

Key words: Compressed Sensing; Information Hiding; Texture Complexity.
\end{abstract}

\section{Introduction}

Information hiding is to embed secret information into the carrier, transmit the secret information through the transmission carrier, this technology has been widely used in the field of information security, because of the hiding of information existence, the attacker can not perceive the existence of secret information. In recent years, the introduction of new theory constantly enrich the innovation of information hiding in the field of technology, compressed sensing (Compressed Sensing CS)[1]theory is very representative, the theory breakthrough the Nyquist theorem of limit, has injected new vitality into the field of information hiding.

In the whole process of compressed sensing, we can get observed value from carrier, the secret information can be hidden in the observations, then use the observation reduction carrier, form a secret information hiding carrier.

Many scholars in this area have made outstanding contributions to the literature [2] proposed using wavelet transform the carrier image transform, then the high frequency part of the image is measured by Gauss observation matrix, the secret information can be hidden in the part of the observation value, achieved a certain effect; the literature [3] is to use the Gauss observation matrix 
all data by wavelet transform on the image after the measurement, the secret information can be hidden in the measurement in this method, the hiding capacity is considerable.

The above algorithm has three problems:

First, the need for the whole image information measurement makes much more time-consuming; Second, observation of the measurements have not been fully utilized, resulting in waste; Thirdly, image imperceptibility can be further improved. This paper proposes a block information capacity of compressed sensing algorithms based on that: according to the secret information capacity and the sampling rate, calculate the required number of observations, the size of the carrier image can be known, the original image is divided into non-overlapping blocks and calculate each image texture complexity, choose the lowest complex texture image block, carry on information hiding by CS technology, and then take the image blocks back into the original carrier image. This algorithm not only saved resources, but also improved the quality of reconstructed image.

\section{Compressed Sensing Technology}

Compressed sensing theory shows that if a signal is sparseness, or in a transform domain is also sparseness, we can use a matrix which is not related to sparse transform as the measurement mapping signals from high dimensional space to low dimension space. The theory not only saves the data storage resources, but also reduces the sampling cost[4].

This theory mainly includes three aspects: sparse transformation, observation matrix, reconstruction algorithm.

\subsection{Sparse transform}

Set the original signal $X=[X(1), X(2), X(3) \ldots X(L)]^{T}$ is a set of one-dimensional discrete signal vector ${ }^{[5]}$, then any group of $\mathrm{L}$ dimensional vector can be showed as $L \times 1$ dimension: $\left\{\psi_{t}\right\}_{t=1}^{L}$,if this vector basis is orthogonal, through $\Psi=\left\{\begin{array}{llll}\psi_{1} & \psi_{t} & \ldots & \psi_{L}\end{array}\right\}$, the original signal can be expressed as:

$$
X=\sum_{t=1}^{L} \beta_{t} \psi_{t} \text { that } X=\Psi \beta
$$

In the formula, $\beta_{t}=\left\langle X, \psi_{t}\right\rangle, X$ is $L \times 1$ dimensional matrix, $\Psi$ is $L \times L$ dimensional matrix, $\beta$ is $L \times 1$ dimension projection coefficient vector on $\Psi$ orthogonal basis by $X$. If there are only $\mathrm{K}(\mathrm{K}<<\mathrm{L})$ larger values (or 
non-zero coefficient) in $\beta$, and the rest of the values are small (or zero), it is said $X$ to be K-sparse, $\Psi$ is the sparse base. Further derived CS formula:

$$
Y=\Phi \Psi \beta
$$

In this formula, $Y$ is observations, $\Phi$ is the observation matrix, $\Psi$ is sparse groups.

\subsection{Observation matrix}

CS theory of the measurement process is to choose an observation matrix to measure the information, so as to get the observation value, the number of observations is less than the original information, so as to play a role in the compression.

We can understand this process as a "morbid equation ". However, not all of the equations can be solved, the theory prove[4], if the observation matrix to meet certain conditions, it can be solved by appropriate algorithm .The solution condition is that the observation matrix satisfies the constraint Restricted Isometry Property (RIP) [6], that is, there is a constant to meet the arbitrary K sparse signal, inequality:

$$
\left(1-\delta_{S}\right)\|\partial\|_{2}^{2} \leq\left\|\Phi_{T} \partial\right\|_{2}^{2} \leq\left(1+\delta_{S}\right)\|\partial\|_{2}^{2}
$$

Establishment, which $T \subset\{1,2 \cdots \mathrm{N}\}$, and $\mathrm{T}<\mathrm{K}$. $\mathrm{M}$ is the number of observations, $\Phi_{T}$ is the sub matrix of the $\mathrm{M} \times \mathrm{T}$ which is constituted column by the index set $\mathrm{T}$ in the observation matrix $\Phi$. From another aspect, it can be understood as follows : the independent of the observation matrix stronger , the effect of the recovery better. So the choice of the observation matrix is very important. Usually, the observation matrix contains random Gauss matrix, Bernoulli matrix, Toeplitz matrix, these matrixs and the sparse base mostly irrelevant, that satisfies the conditions.

\subsection{Reconstruction Algorithm}

The current solution algorithm is greedy pursuit algorithm, each iteration to select a local optimal solution to approximate the original signal. Including matching pursuit (MP)[7] and orthogonal matching pursuit (OMP)[8] algorithm. This kind of algorithm is much faster, but it needs much more observed value. There is a convex relaxation algorithm, the algorithm is to transform the non convex optimization problem into a convex optimization problem, and obtain the approximation of the original signal. Representative algorithm: Base tracking algorithm, this kind of algorithm needs less observation, but the computational complexity is larger. The idea method is OMP algorithm, which 
is an iterative greedy algorithm, each iteration and iterative margin with the biggest correlation atoms (the measurement matrix $\Phi$ column index), join the last iteration of the support set, then the iterative residual update, namely from the measured value of $\mathrm{Y}$ minus the orthogonal projection in the selected atom the number of iterations until is equal to the sparsity of $\mathrm{K}$ signal, the iteration is stopped. OMP algorithm is more suitable for image reconstruction when the amount of signal data is large, the algorithm can be used to reduce the amount of computation.

\section{Block by Using Image Texture Information}

Texture is the description of the spatial distribution pattern of the pixel gray level of the image, which reflects the quality of the image, such as roughness, smoothness, granularity, randomness and standardization. Image texture complexity is usually measured by using standard deviation .In the image, each pixel $\mathrm{f}(\mathrm{i}, \mathrm{j}) 3 \times 3$ of the standard deviation is defined as:

$$
S_{s t d}=\sqrt{\frac{1}{9} \sum_{m=-1}^{1} \sum_{n=-1}^{1}(f(i+m, j+n)-\bar{f})^{2}}
$$

$\bar{f}$ expressed in $f(i, j)$ as the center of the average value of $3 \times 3$ pixels, Sstd expressed $f(i, j)$ standard deviation. The texture complexity of the block image can be expressed as

$$
T c=\frac{1}{m \times n} \sum_{i=1}^{m} \sum_{j=1}^{n} S_{s t d}(i, j)
$$

Tc represents the texture complexity of the block image, $n, m$ expressed the pixel size of the image block.

The image texture is different as it contains the amount of information. It used for processing of compressed sensing technology, the effects are different. When the image texture is smooth, with few observations can get ideal effect, but when the image texture is more complicated, even more observation value is difficult to accurately reconstruct the information [9].

The image restoration effect can be measured by the peak signal to noise ratio (PSNR)[10], the higher the PSNR is, the better the recovery effect is, and the calculation formula is as follows:

$$
P S N R=10 \log \left(\frac{255^{2}}{\frac{1}{M \times N} \sum_{i=0}^{M-1} \sum_{j=0}^{N-1}\left[X(i, j)-X^{\prime}(i, j)\right]^{2}}\right)
$$


In the formula, $\mathrm{X}$ as the original carrier image, $\mathrm{X}$ 'as the carrier density image, $\mathrm{m}, \mathrm{n}$ for the number of rows and columns of pixels. When the PSNR is greater than $30 \mathrm{~dB}$, it will not cause the sensitive response of human vision, the restoration effect is ideal.

The following image "Lena" was used to test, which size is $256 \times 256$. The image was divided into 16 blocks ,each of them is $64 \times 64$.It was numbered as from left to right, from top to bottom, calculate each texture complexity, and the sampling rate for CS treatment is 0.6 , calculate the reduction of image blocks and the original image block PSNR, as shown in the following table:

Tab. 1 Comparison of texture complexity and peak signal to noise ratio

\begin{tabular}{cccccc}
\hline Number & $\begin{array}{c}\text { Texture } \\
\text { complexity }\end{array}$ & $\begin{array}{c}\text { Peak signal to } \\
\text { noise ratio }(\mathrm{dB}) \\
\text { number } \\
(\mathrm{dB})\end{array}$ & Number & $\begin{array}{c}\text { Texture } \\
\text { complexity }\end{array}$ & $\begin{array}{c}\text { Peak signal to } \\
\text { noise ratio(dB) }\end{array}$ \\
\hline 1 & 6.59 & 26.43 & 9 & 15.40 & 23.54 \\
2 & 9.00 & 25.23 & 10 & 20.58 & 22.12 \\
3 & 7.92 & 25.90 & 11 & 15.41 & 23.24 \\
4 & 10.42 & 24.06 & 12 & 5.94 & 26.50 \\
5 & 8.79 & 25.50 & 13 & 16.20 & 23.51 \\
6 & 18.78 & 22.64 & 14 & 17.15 & 22.94 \\
7 & 16.87 & 21.58 & 15 & 10.23 & 24.04 \\
8 & 8.30 & 25.45 & 16 & 10.80 & 24.01 \\
\hline
\end{tabular}

Through tests, it can conclude that the higher $T c$ value, the worse the reduction of image effect with CS technology.

The secret information can be hidden in the lower value of the image block Tc, the entire image imperceptibility is ideal and the security of the information has also been improved.

Based on the testing, in this paper, we try to select the region with smooth texture to CS processing and information hiding in order to ensure that the image is much better.

\section{Algorithm in this Paper}

\subsection{Secret information hiding}

The secret information hiding mainly includes the following four steps:

(1) According to the number of secret information which needed to hide and the sample rate, choose the appropriate size of the carrier image block, and in accordance with this carrier block, which does not overlap; 
(2) Calculate the texture complexity of the image block, select the image block with the smallest texture complexity and this is the carrier of the secret information;

(3) Sparse transform of the image block, and measured by the observation matrix $\Phi$, it get the observations. Write the secret information into the observations, and then restore the carrier block image. This is the stego image block;

(4) Put the image back to the original position, restore the entire image, finish information hiding.

The hidden process is as follows

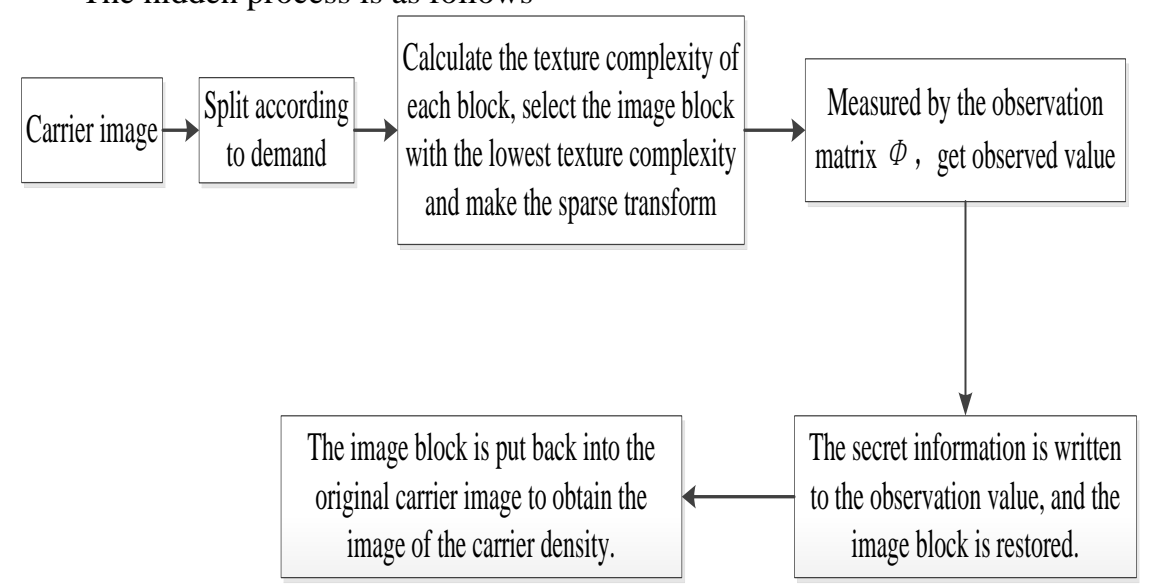

Fig. 1 Information hiding process

\subsection{Extraction of secret information}

After receiving the information, the secret information needs to be extracted. The extraction of the secret information includes the following three steps:

(1) Do the same block segmentation of the original image and the transmission of the image, and calculate the texture complexity of each image of the original image;

(2) Select the image block with the smallest texture complexity, record its position as $n$, and select the same position $n$ block image in the carrier density image, which is the image of the carrier;

(3) Processing two images with the same CS as the transmitter, and get the two sets of observation values.It can be used to extract the secret information.

Its extraction process is as follows: 


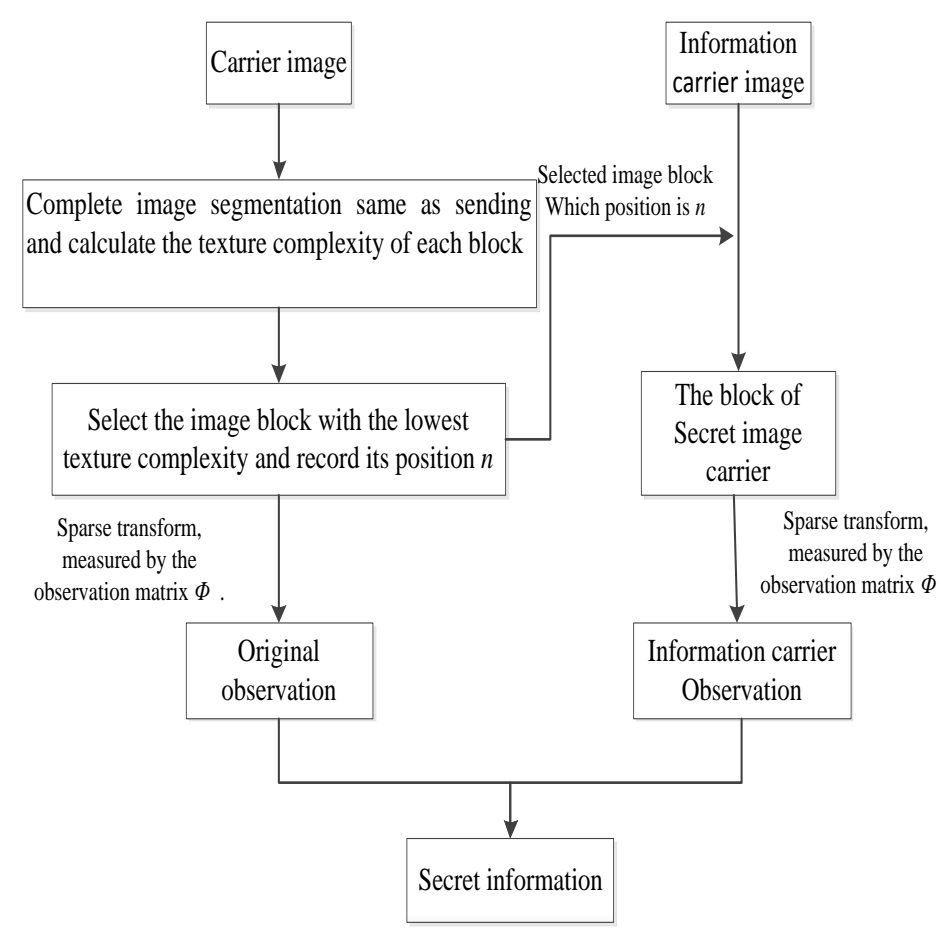

Fig. 2 Information extraction process

\section{Simulation Test}

It used MATLAB software for the experiment of this algorithm , the two value image digital “数字水印”, its size is “64 × 64", the gray image "Woman", "Lena", "Lion" as the carrier, their size are all " $256 \times 256$ ". For convenience, the carrier image is divided into 16 blocks, each block size is "64 $\times 64$ ". After sparse transform, it takes sample by 1:1. Each observation vector image values obtained with the same number of secret information, so each carrier image exactly can completely hide the secret information.

It can describe the problem clearly. With this algorithm, the results as follows: 


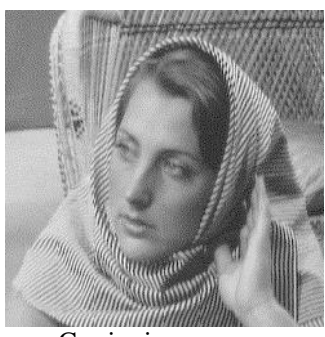

Carrier image

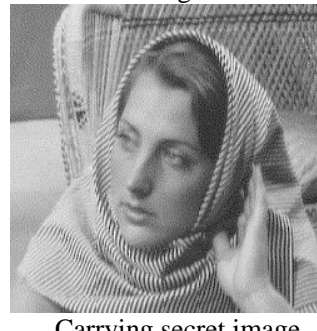

Carrying secret image

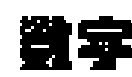

KED

Secret information

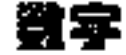

㫤

Extract information

Fig. 3 Part of the experimental results

The result is ideal from the visual effect, it would analysis by mathematics method on success.

\subsection{Transparency analysis}

Transparency refers to the image information hiding which can be unperceived, the higher degree of transparency, the more perfect. It used peak signal to noise ratio (PSNR) commonly to measure the traditional algorithm and the algorithm of this paper, the results are as follows:

Tab. 2 The PSNR comparison of traditional algorithm and this algorithm

\begin{tabular}{ccccc}
\hline $\begin{array}{c}\text { Image } \\
\text { name }\end{array}$ & $\begin{array}{c}\text { Image block number with } \\
\text { minimum } T c \text { value }\end{array}$ & $\begin{array}{c}\text { Traditional algorithm } \\
\text { PSNR(dB) }\end{array}$ & $\begin{array}{c}\text { Algorithm in this } \\
\text { paper PSNR(dB) }\end{array}$ & $\begin{array}{c}T c \\
\text { value }\end{array}$ \\
\hline Woman & 9 & 35.60 & 37.11 & 10.30 \\
Lena & 12 & 34.31 & 36.95 & 5.95 \\
Lion & 7 & 32.95 & 35.62 & 9.45 \\
\hline
\end{tabular}

Part of the test results are as follows: 


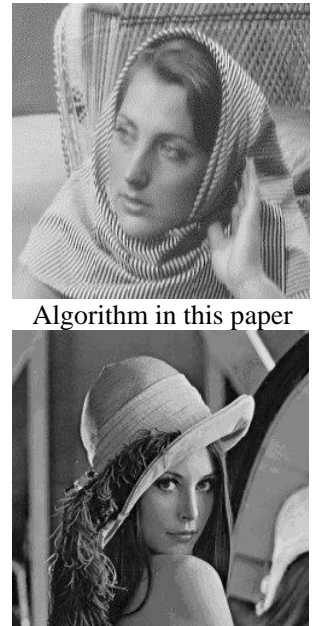

Algorithm in this paper

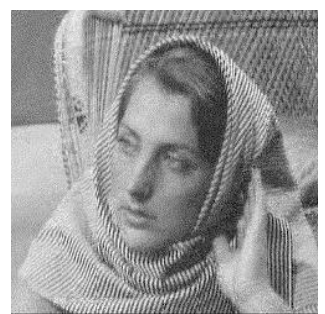

Tradition alalgorithm

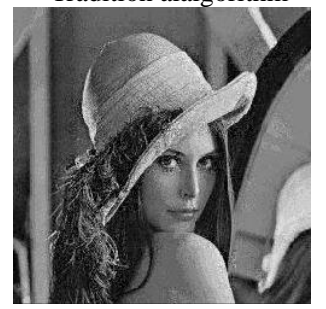

Traditional algorithm

Fig. 4 Part of the information hiding results

It completed information hiding by using the traditional algorithm, the whole carrier image used by CS processing, the higher complexity texture part of the recovery effect is not ideal, so it influenced the hidden effect. This algorithm is only use part of image which complexity texture is the lowest completed CS transform and hidden secret information. The transparency is better than the traditional algorithm .Through the test data and the results, it demonstrate the superiority of the proposed algorithm.

\subsection{Analysis of extraction quality}

The extraction effect of this algorithm is shown as follows:

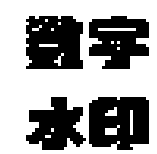

Original information

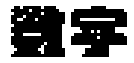

KED

Extracted information

The extraction effect is ideal by visual perspective. The commonly method is to calculate the normalized correlation (NC value) of the two images .The calculation formula is as follows: 


$$
N C=\frac{\sum_{i=0}^{L-1} \sum_{j=0}^{K-1} x(i, j) x^{\prime}(i, j)}{\sum_{i=0}^{L-1} \sum_{K=0}^{K-1}[x(i, j)]^{2}}
$$

$x$ is the original secret information, $x$ 'is the extracting secret information, $L$, $K$ are the numbers of pixels ranks and columns, respectively. When the NC value is less than 0.5 , extraction failure. In this paper, the algorithm NC value is up to 0.9917 , it satisfy the requirements of information extraction.

\subsection{Analysis of consuming time}

The traditional algorithm consume more time, because it need to calculate the total observation value of the whole carrier. This algorithm only select the low complex texture image blocks for secret information hiding, it can saves resources. The experiment using three images of the two algorithms to calculate the complete time, the result as shown :

Tab. 3 The time consuming comparison of traditional algorithm and this algorithm

\begin{tabular}{ccc}
\hline Name & Traditional algorithm consume time(s) & This algorithm consume time(s) \\
\hline Woman & 267.5 & 5.87 \\
Lena & 268.9 & 5.92 \\
Lion & 265.3 & 5.81 \\
\hline
\end{tabular}

The table show that the algorithm saves a lot of time, and improve efficiency.

\subsection{Robustness analysis}

The confidential information is subject to varying degrees of interference, such as Gauss noise, salt and pepper noise in the transmission process .In the experiment, we add this two kinds of noise to the carrier in order to test the robustness of the algorithm .With different strength, it calculate the NC value of the original secret information, the results are as follows:

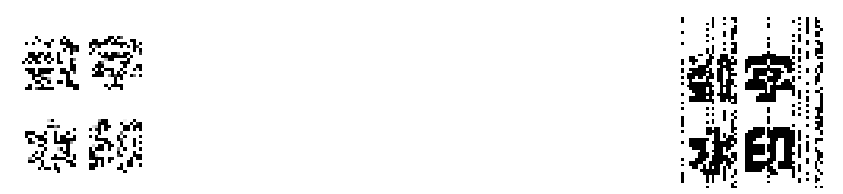

Gauss noise variance 0.001 (NC: 0.89 )

Salt and pepper noise intensity 0.002 (NC: 0.7213 )

Fig. 6 Extraction effect under certain noise

From the experimental results, we can summarizes that although the noise 
has a certain effect on the secret information, it still can understand the contents of the secret information, the algorithm of this paper has a certain robustness.

\subsection{Anti extraction analysis}

During the information transmission process, it may be intercepted by the eavesdrops in order to extract the secret information, if you do not master the observation matrix, it is impossible to get information, if it use another observation matrix, just like this:

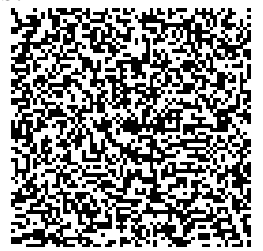

Fig. 7 Extraction effect without the correct Observation matrix

The effect is completely beyond recognition, so the algorithm of this paper ensures the security of the secret information.

\section{Conclusion}

The traditional method is to find the large hidden redundant space, the secret information can be hidden in it, but the redundant space itself is lack of consideration, these algorithms cause different degrees of waste of resources; This paper has overcome the traditional thinking, the "tailored", according to the number of secret information, calculate the appropriate redundancy of the carrier, selection ideal block and hiding information. The method save computing resources, improve imperceptibility and robustness, at last extraction resistance. This algorithm needs to be further strengthened in the anti noise attack.

\section{Reference}

1. Candes E, Romberg J,Tao T.Robust uncertainty principles:exact signal reconstruction from highly incomplete frequency information[J].IEEE Transactions on Information Theory,2006,52(2):489-509.

2. CHEN Guo-fa,GUO Shu-xu,LI Yang,LI Liang,Digital image watermark algorithm based on compressive sensing. Modern Electronics Technique, Vol 35(2012),pp:98-100.(In Chinese).

3. WEI Feng, LIANG Dong, ZHANG Cheng, Watermarking algorithm for 
digital image based on compressive sensing measurements. Journal of Anhui University(Natural Sciences), Vol 37(2013),pp:61-68. (In Chinese).

4. REN Yue-Mei, ZHANG Yan-Ning, LI Ying, Advances and Perspective on Compressed Sensing and Application on Image Processing, Acta Automatica Sinica,Vol.40(2014),pp: 1563-1575. (In Chinese).

5. Li Shu-xing, Ge Gen-nian.Deterministic construction sparse sensing matrices via finite geometry.IEEE Transactions on Information Theory, Vol.62(2014): pp 2850- 2859.

6. Candes E, Romberg J, Sparsity and incoherence in compressive sampling. Inverse Problems, Vol.23 (2007),pp :969-985.

7. LIU Fang,WU Jiao, YANG Shu-Yuan, JIAO Li-Cheng, Research Advances on Structured Compre-ssive Sensing. Acta Automatica Sinica, Vol.39(2013),pp:1980-1995. (In Chinese).

8. RONG Yan-xia,QIU Xiao-hui, Image Blocking Compressed Sensing Algorithm Based on Wavelet Transform. Comnputer Technology and Development, Vol.25(2015),pp:29-32. (In Chinese).

9. Cao Yuqiang,Bai Sen,Cao Mingwu,Image compression sampling based on adaptive block compressed sensing. Journal of Image and Graphics, Vol.21(2016),pp:416-424. (In Chinese).

10. WANG Zhi- ren,ZHANG De- sheng,NIE Dong- dong, Construction of Measurement Matrix in Compressed Sensing via Logistic Chaos Sequence. Journal of Chinese Computer Syste ms, Vol.37 (2016), pp:588-592. (In Chinese). 